\title{
BMJ Open Research protocol for the exploration of experiences of Aboriginal Australian mothers and healthcare professionals when using the Edinburgh Postnatal Depression Scale: a process-oriented validation study using triangulated participatory mixed methods
}

\author{
Ai Wen Chan, ${ }^{1}$ Petra Skeffington, ${ }^{2,3}$ Corinne Reid, ${ }^{3,4}$ Rhonda Marriott ${ }^{3}$
}

To cite: Chan AW, Skeffington $P$, Reid C, et al. Research protocol for the exploration of experiences of Aboriginal Australian mothers and healthcare professionals when using the Edinburgh Postnatal Depression Scale: a processoriented validation study using triangulated participatory mixed methods. BMJ Open 2018;8:e022273. doi:10.1136/ bmjopen-2018-022273

- Prepublication history for this paper is available online. To view these files, please visit the journal online (http://dx.doi. org/10.1136/bmjopen-2018022273).

Received 10 February 2018 Revised 19 June 2018 Accepted 4 August 2018

A) Check for updates

(C) Author(s) (or their employer(s)) 2018. Re-use permitted under CC BY-NC. No commercial re-use. See rights and permissions. Published by BMJ.

For numbered affiliations see end of article.

Correspondence to

Ai Wen Chan;

A.Chan@murdoch.edu.au

\section{ABSTRACT}

Introduction Adopting a process-oriented framework for test validation can help to establish whether this tool has the potential to be an acceptable, valid and reliable indicator of depression for mothers and mothers-to-be. This mixed-methods research protocol seeks to explore the views and experiences of Aboriginal mothers and healthcare professionals in relation to the Edinburgh Postnatal Depression Scale (EPDS), and is intended to highlight potential barriers in perinatal mental health conceptualisation, engagement and response style. Methods and analysis Thematic analysis will be applied to interview transcripts of Aboriginal Australian mothers $(n=6+)$ and healthcare professionals $(n=6+)$ to identify key themes. The process-focused validation model will use narratives about experiences of using the EPDS as the priority point of analysis. Item-level data and process-level (experience) data are key phenomenological data. The interview-based narratives will be then compared with EPDS scores to check for points of congruence and divergence. This will be done at two time points, antenatally and postnatally, to assess changes in assessment processes and perceptions. Bridging evidence-based research with clinical practice in an Aboriginal Australian context will be facilitated by an investigation of the perceived cultural relevance and likely clinical effectiveness of EPDS. Such evidence is critical to understanding whether the EPDS fulfils its intended purpose. The guiding principles in designing this research protocol is to benefit the well-being of young Aboriginal families and communities through partnership with Aboriginal women.

Ethics and dissemination Ethics approval was obtained from Human Research Ethics Committee of Murdoch University and from Western Australian Aboriginal Health Ethics Committee (WAAHEC). Participating healthcare sites and services have provided letters of support. Results of this study will be submitted for publication in a peerreviewed journal.
Strengths and limitations of this study

- Triangulation of data will strengthen confidence in the findings and recommendations for future use, adaptation or replacement of the Edinburgh Postnatal Depression Scale (EPDS) measure in this context.

- Adapting research design by incorporating Indigenous methodologies with more 'mainstream' research methodologies to better understand mental health within an Aboriginal cultural context.

- Adopting a more process-oriented approach, to extend findings from traditional validation approaches.

- Aboriginal cultures are not homogeneous in terms of culture and language; this study will be collecting data from Western Australia region, so a compromise of the depth of this study is its generalisability.

- This mixed-methods design is preliminary, but has the potential to encourage further investigation of psychometric properties, predictive validity and reliability, of the EPDS tool when used within an Aboriginal Australian context.

\section{BACKGROUND}

It is common for those stepping into new roles as parents to experience stress and anxiety, even if they have had parenting experience with previous children. When the combination of hormonal changes and overwhelming parental responsibilities begin to subside, a parent's mood and emotional changes typically start to resolve and re-stabilise. However, if realities of parenthood do not match the expectations or resources, a range of emotions, feelings and behavioural changes such as anxiety, sadness, irritability, constant worrying, guilt, lack of interest in 
baby, changes in eating and sleeping habits, thoughts of hopelessness and worthlessness, and feeling of being inadequate or incompetent to cope with the newborn baby may consolidate or even deepen. ${ }^{1}$

Results of the 2010 Australian National Infant Feeding Survey data showed that one in five mothers of children aged 24 months or less were diagnosed with depression, with more than half of these mothers noting that their diagnosed depression was during the perinatal period. ${ }^{2}$ In other words, an estimated 111000 mothers were diagnosed with depression during 2010-2011, of which 56000 were with perinatal onset. ${ }^{2}$ However, Halbreich and Karkun ${ }^{3}$ found that there is cultural variability in the reported prevalence rates of postnatal depression. Based on evidence of multiple and complex risk factors, ${ }^{4}$ the prevalence of perinatal depression for Aboriginal ${ }^{\mathrm{i}}$ women is thought to be higher than for non-Aboriginal ${ }^{\mathrm{ii}}$ women. ${ }^{56}$ However, errors in estimated prevalence rates are likely due to women's ambivalence and concern around engaging with government services and a consequent reluctance to seek support, thus resulting in under-representation in key datasets. ${ }^{7}$ The factors driving this ambivalence may also be reflected in communication and trust when completing perinatal mental health screening tests. ${ }^{8}$ This study will contribute to the growing evidence base for addressing access barriers for Aboriginal mothers by considering the experience of Aboriginal mothers and healthcare professionals when engaging with routine perinatal mental health screening processes.

\section{Importance of screening}

Being submerged in significant stress responses for an extended period can put parents at risk of developing new, or exacerbating current, mental health issues during the perinatal period. ${ }^{9}$ In turn, perinatal mental health complications can have cascading and lasting impacts on the child and family and has been deemed a global health concern, particularly in disadvantaged communities and developing countries ${ }^{10}{ }^{11}$ In Australia, Aboriginal women have more vulnerable reproductive and maternal health trajectories compared with other Australian women, ${ }^{12}$ which may put them at higher risk for poor mental health outcomes. Reports consistently indicate more perinatal deaths ${ }^{13}$ and higher infant mortality, infants being more likely to die during or soon after childbirth. ${ }^{14-16}$ Aboriginal Australian women are known to be three times more likely to smoke during pregnancy, more than twice as likely to have a baby of low birth weight (weight at

${ }^{i}$ It is acknowledged that different Aboriginal groups prefer different terminology. The term 'Aboriginal' is used respectfully in this paper in reference to persons who identify themselves as Aboriginal and/or Torres Strait Islanders descent, unless otherwise stated. It is not an indication of disrespect to Torres Strait Islander people. http://www.healthinfonet.ecu.edu.au/uploads/resources/30201_30201.pdf

${ }^{i i}$ Unless a direct quote from a source, this paper will hereafter respectfully use the term 'Aboriginal'. It is not an indication of disrespect to Torres Strait Islander people. birth $<2500 \mathrm{~g}$ ) and to have more children being born prematurely (prior to 37 weeks gestation). ${ }^{141617}$

Research shows that perinatal depression can compromise the emotional and mental well-being of not only the mother herself, ${ }^{18}$ but can affect those around her including her partner's health and well-being, ${ }^{19}$ and the infant's sense of attachment and development. ${ }^{20}{ }^{21}$ If at-risk mothers are identified at an early stage, antenatal care and supported programmes can provide opportunities for positive outcomes associated with healthy infant birth weight, stronger maternal nutrition and health and better outcomes for the mother's relationships with her infant and her partner. ${ }^{22}{ }^{23}$ Support is especially critical during the formative and foundational early years of infant's development, a fact recognised in recent federal government health initiatives. ${ }^{19}$ While there is a broad understanding of Aboriginal social and emotional wellbeing (SEWB) and health outcomes, there is a clear gap in the investigation of experiences, perceptions and concerns around perinatal mental health screening and the cultural acceptability of current perinatal mental health services.

\section{Edinburgh Postnatal Depression Scale}

Originally, the Edinburgh Postnatal Depression Scale (EPDS) was developed to identify women who were at risk of depression during the postnatal period. ${ }^{24}$ Based on most recent practice guidelines, latest research and consultations with perinatal mental health specialists and representatives in Western Australia, ${ }^{25}$ it is clear that the EPDS is offered during both the antenatal and postnatal period when possible. ${ }^{24-29}$ While the EPDS is nationally and internationally considered gold standard practice for perinatal mental health screening, the use of EPDS with Aboriginal Australians has received little attention in current research and practice. There is also a lack of alternative options that are culturally informed. ${ }^{30}$

Recent Australian research has identified cultural differences in traditional health beliefs and conceptualisation of mental health ${ }^{31} 32$; perception and interpretation of language meanings and its consequential effect on communication; and beliefs around the cultural relevance of Western psychotherapy. ${ }^{33} 34$ More specifically, research around the use of the EPDS with Aboriginal women has indicated that the language used in the EPDS is often misinterpreted. ${ }^{20}$ Moreover, healthcare providers find it challenging to use the EPDS in this context as they report that Aboriginal women feel uncomfortable completing the questionnaire and untrusting of the intent of the screening process. ${ }^{35}$ Cultural differences have been acknowledged, but has not been meaningfully or comprehensively investigated. Hence, the translation of these preliminary research findings into better routine practice for Aboriginal mothers is lacking.

\section{Barriers to engaging with EPDS screening}

There has been a lack of research to help better understand the barriers, or the potential, of using the EPDS 
with Aboriginal women. Difficulties around literacy, language and communication create potential barriers to engaging with the EPDS screening process in some cases. ${ }^{32} 36$ However, existing research and literature also underlines the primary importance of cultural and historical influences on Aboriginal women's engagement with health services and how this may impact the way healthcare professionals engage with Aboriginal mothers.

In 2006, the Western Australia Perinatal Mental Health Unit investigated some of the factors impacting accessibility of services. ${ }^{37}$ Results demonstrated that Aboriginal mothers were reluctant to seek professional support because of the mistrust and fear attached to accessing services, while healthcare professionals did not provide actions considered culturally safe if, or when, an Aboriginal mother presented for help. This report highlighted the need for improved engagement that fosters culturally safe relationships with services for Aboriginal women.

The history of colonisation provides an important context here. It has contributed to the mistrust of government services, deeply held fears of being judged as an inadequate parent and a realistic fear that children may be removed. ${ }^{38}$ In 2012-2013, an estimated $16 \%$ of Aboriginal people (aged 15 and older) reported being removed from their natural family and $48 \%$ had relatives who had been removed from their natural family. ${ }^{6}$ Forced removal has had a subsequent intergenerational impact on the SEWB of today's Aboriginal people and communities. This and other unsatisfactory and traumatic experiences have, in turn, resulted in a significant degree of fear when accessing mainstream healthcare services. ${ }^{39} 40$ This feeling of mistrust and fear of government authorities can make Aboriginal people reluctant to engage with services and wary of providing personal information on which unintended decisions may have been made. ${ }^{41}$ Hence, Aboriginal women with perinatal depression may remain disproportionately undetected and untreated, highlighting the importance of accessible and culturally congruent screening to support identification of symptoms of depression in the perinatal period. ${ }^{41}$

\section{Culturally congruent screening}

To date, there have been only two published evaluations of the EPDS used within the Aboriginal cultural context. One study involved the development of a translated version of the EPDS in community-controlled Townsville Aboriginal and Islander Health Service (TAIHS) and at Mt Isa. ${ }^{42}$ The reliability of the TAIHS EPDS was compared against the standard EPDS with correlational analyses to investigate internal consistency and item-total correlation. Comparable levels of internal consistency suggested that the scales measured the same construct in a reliable way. However, the small number of participants who were able to complete both the TAIHS EPDS and the standard EPDS antenatally $(\mathrm{n}=94)$ and postnatally $(\mathrm{n}=50)$ constrained confidence in the internal consistency and power analysis. Further, the lack of concurrent validity was acknowledged as a significant limitation. ${ }^{30} 4243$
Even though no comprehensive analysis of psychometric properties resulted from the study, researchers found that Aboriginal women wanted to continue being involved in the study as they were able to use their 'own' screening materials [42, p. 130]. More work in this area was encouraged to investigate and further evaluate the translated version of the EPDS.

The TAIHS EPDS was utilised again in later research with a purposive sample of 96 Aboriginal women to investigate the impact of social stressors and emotional mental health among pregnant Aboriginal women. ${ }^{30}$ High scores on the TAIHS EPDS positively correlated with mothers' history of child abuse and a history of exposure to domestic violence. ${ }^{30}$ The process of developing the TAIHS EPDS demonstrated the potential for usefully modifying the EPDS. However, further evaluation of the TAIHS EPDS instrument to establish validity and reliability would require significant resource investment for limited application as the adapted language was specific to a remote setting in Townsville. Aboriginal languages differ among the vastly diverse Aboriginal communities; ${ }^{43}$ therefore, the TAIHS EPDS version could not be generalised to the wider Aboriginal community. Further investment and research in perinatal mental health screening needs to prioritise generalisability.

Another research group developed a culturally relevant screening tool as an alternative version of the EPDS through a collaboration with Kimberley healthcare providers and over 100 Aboriginal women. This tool is called the Kimberley Mums Mood Scale (KMMS). ${ }^{36}$ The KMMS was deemed (i) reliable, with a reasonable Cronbach's alpha of 0.89 , and (ii) valid through comparison of scores to blinded diagnosis by a clinical expert who conducted a semistructured clinical interview with each participant to determine diagnosis and immediate service needed. This is now the preferred version of the EPDS in the Kimberley region in Western Australia. ${ }^{44}$ However, again, the main limitation of the study was having a small convenience sample from a specific region in remote Western Australia, limiting generalisability. ${ }^{44}$

With the exception of these two study areas, the EPDS has been, and continues to be, administered with Aboriginal Australians as routine practice. ${ }^{25}$ Both studies have their limitations but both have centred on the view that the gold standard version of the EPDS may not work effectively with Aboriginal clients. Further, that Aboriginal women seem ambivalent in their engagement with the EPDS, ${ }^{36}$ possibly because of linguistic, cultural and practical factors that could create potential barriers in engagement and responsiveness to survey items. ${ }^{44}$ Subsequent to this, reluctance to seek services post screening may shed light on possible under-detection and misrepresentation of mental health issues among Aboriginal women; and therefore, could be left untreated. Clearly, there remains a clinical need to explore and confirm which screening processes and tools are most culturally sensitive and effective in identifying women who would benefit from perinatal mental health support. 


\section{The current study: narrowing the gap}

Cultural, social and health gaps between Aboriginal and non-Aboriginal Australians are persistent and need to be addressed to support strong families and communities. ${ }^{45}$ There is long-standing evidence of compromised perinatal mental health outcomes for Aboriginal mothers (although likely under-estimated) and its consequential impacts on families. As there is no screening tool for perinatal depression that is adequately validated in the Aboriginal context, a close examination of the EPDS focused on the process and the context of its use with Aboriginal women is imperative to laying foundations for future screening development. Exploring, and appreciating, the strengths and limitations of the EPDS when used in this context can highlight areas of need and potential improvements.

The current study aims to collaborate with Aboriginal mothers and healthcare professionals to explore perinatal mental health screening experiences using a mixed-methods participatory design ${ }^{46}$ to undertake a process-focused approach to screening validation. ${ }^{47}$ This research draws attention to the step towards acquiring knowledge of Aboriginal individuals and communities to promote empowering ownership of that knowledge, and away from the domination and oppression that research, and it's scientific scrutiny may unintentionally play a role in. ${ }^{48}$ According to Rigney [48, p 116], who coined the term Indigenist research, follows three fundamental principles: (a) involvement in resistance as the emancipator imperative; (b) political integrity; and (c) providing privilege to Indigenous voices. This research protocol adopts Aboriginal research methodologies that facilitate these three core principles in order to engage in participatory community-driven and research-to-practice approach that is more translational and relevant to the Aboriginal Australians.

\section{RESEARCH PROTOCOL}

\section{Objectives}

Seeing as the EPDS is currently freely available and the recommended screening tool in the perinatal period, there is a clear clinical imperative to explore whether or not the process and context of administering and completing the EPDS meets the cultural needs of Aboriginal people.

A common criticism by Aboriginal Australians is that communities are not consulted until after decisions are made about the development and implementation of programmes [7p7] .Thus, this study will adopt a Patient and Public Involvement (PPI) model $^{46}$ that prioritises Aboriginal mothers and perinatal healthcare professionals as partners in evaluating this critical period of engagement with health services. To ensure contextual richness, a mixed methods approach will be adopted to evaluate the routine administration of the EPDS as the gold standard perinatal mental health screening tool (EPDS).
This research protocol will also adopt a process-oriented screening validation approach, ${ }^{47}$ which shifts the emphasis towards process research. EPDS scores (itemlevel data) and narratives and experiences of using the EPDS (process-level data) will both be used, but the latter will be the priority point of analysis to capture the psychological processes during screening. It is in this context that this research aims to explore personal views and experiences of Aboriginal mothers when using the EPDS. It emphasises the importance of understanding the context of delivering and applying screening in the perinatal period (EPDS), which could potentially facilitate and complement future investigation around the diagnostic and predictive validity and likely reliability of this instrument when used with Aboriginal mothers. This study includes views about the instrument itself and also how healthcare professional's administration can intentionally and unintentionally influence how the EPDS is perceived and used. Therefore, this research protocol investigates the application of the EPDS within the context of Aboriginal populations by prioritising the experiences of EPDS users, which includes Aboriginal mothers and healthcare professionals (whom in the main are not Aboriginal). ${ }^{49}$

This research seeks to answer the following questions:

1. The EPDS is used nationally and internationally, but is it clinically effective and culturally congruent when applied with Australian Aboriginal populations?

2. Are the responses to EPDS items, and changes in EPDS scores over time, consistent with the narratives of women experiencing the EPDS? Do the experiences of using the EPDS reflect and match the EPDS responses?

3. A client may have either an unpleasant or pleasant experience during the first screening session. Do these experiences influence or change with repeat screening?

4. What are the experiences of Aboriginal mothers when they interact with the EPDS? How do Aboriginal mothers perceive and/or receive the administration of the EPDS? How do Aboriginal mothers perceive the relevance of the items to understanding how they are feeling or coping? How do mothers understand or interpret the severity rating scales?

5 . What are the experiences of midwives and healthcare professionals when they administer the EPDS with Aboriginal mothers?

Findings may support current practice. Alternately, findings may support adaptation to content or screening processes, or may indicate a need for replacement of the EPDS with a new tool. As a preliminary exploratory study, this proposed research creates a strong methodological template of knowledge translation $^{50}$ and participant engagement ${ }^{46}$ as integral parts of the process to support systemic modifications in routine screening processes to improve SEWB for Aboriginal families and communities, and indeed, when planning other healthcare modifications to meet the needs of special groups. 


\section{Method}

\section{Study design}

Ensuring cultural congruence and engaging in reciprocal relationships is vital when working in a cross-cultural context. ${ }^{51}$ We aim to achieve this through regular and iterative consultation with an Aboriginal Advisory Group $^{\text {iii }}$ to ensure cultural integrity, security and cultural endorsement in this process. Consultation with an Aboriginal Advisory Group informs collaborative community inquiry that is necessary to engage in social research and change. $^{52}$

Rather than a traditional psychometric validation study, this preliminary research project will adopt a process-oriented validation model and use a triangulation design to evaluate whether the EPDS is suitable for use in an Aboriginal Australian context. Thus, this study will adopt an exploratory participatory mixed-methods research design. ${ }^{46} 53$ This requires gathering data from different sources, and incorporating different perspectives to help researchers gain deeper understanding. ${ }^{54}$ Qualitative analysis will focus on interview data targeting participants' personal and contextual experiences when interacting with the EPDS screening tool and screening process. The completion of the EPDS and interviews will be undertaken at two different time-points - once antenatally (at minimum of 10-16 weeks pregnant) and once postnatally (6-8 weeks following birth). Qualitative and quantitative data will be triangulated to see whether perceptions and experiences of well-being and depression equate across EPDS responses and interview data.

A yarning interview methodology will be adopted as it has been established as being the most culturally congruent means of interview from an Aboriginal perspective. $^{56}$ Individuals' experiences and cultural points of views are critical for enabling translational research. Aboriginal families and communities who are participating in research should be given a safe space to voice and yarn about their experiences without judgement. ${ }^{57} 58$ In the proposed study, 'yarning'/storytelling interview sessions will be commenced by an Aboriginal cultural consultant who is familiar and has close ties with the Aboriginal community. Yarning sessions follow a patient-centred approach, which can facilitate a safe and secure environment for successful communication between Aboriginal clients and the Aboriginal cultural consultant. $^{56}$

Qualitative data will be analysed thematically using a process of data immersion, data coding, creation of categories and identification of themes. ${ }^{59}$ Data immersion will involve listening to the digital recordings, making corrections to the transcripts and reading and re-reading the transcripts. Through data immersion, key

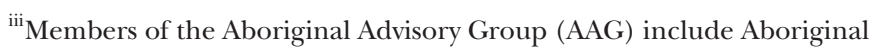
community Elders, seniors, men and women. AAG members are a part of a larger study that this research protocol will assist.
}

characteristics and in-depth meanings and descriptions of experiences will emerge through the categorisation and creation of themes. A systematic process of data coding involves developing and differentiating codes under potential themes or subthemes. While grasping the essence and nature of emerging themes, the outcomes of coding will be scrutinised for recurrence and iteration. ${ }^{60}$

An alternative approach to coding and thematic classification is the case-focused approach where narratives (profiles) are contextualised from interviews and re-integrated into analysis. ${ }^{61}$ Rather than simply a presentation or a description of a case study, categorised data (codes) can occur in a unique context of a particular case-rather than between cases. Furthermore, contextualising narratives recognises the importance of diversity, rather than being explained by codes and reductive formulations. Connecting categorised codes with case-focused narratives ('profiles') can yield more themes or sub-themes, which can then be seen in relation to others' themes and already established knowledge.

Quantitative data will be collected through EPDS completion. Consistent with a process-oriented validation approach, it should be noted that the primary interest is not the EPDS score per se but how women arrive at their responses. For example, are the rating scales used in a differentiating way? Are there patterns in response style for any particular items? Do changes in response over time reflect personal change as revealed in the interview?

Quantitative data will be recorded on Excel, while the qualitative data analysis will be conducted with NVivo. As data emerges in an iterative and cyclical manner, linking and merging data during interpretation and analysis of themes would have to be considered. Data triangulation is required to draw together both quantitative and qualitative data to provide a richer and more comprehensive answer to this unique research question. ${ }^{62}$

\section{Participants}

Researchers will invite participation from members of nominated participating organisations, including local hospitals and community health services in Western Australia. Eligible participants are mothers who identify themselves as being of Aboriginal and/or Torres Strait Islander descent; those who are at least 10-16 weeks pregnant are deemed eligible. Aboriginal mothers are not eligible if they report current or recent (within the past 6 months) experiences of significant self-harm or trauma, or are diagnosed with a mental illness, or were previously wards of the state. This will be identified by the healthcare professionals from the elected participatory sites. Participants will be followed up by these referring healthcare professionals if clients are deemed to be experiencing significant self-harm or trauma at any time.

In addition, healthcare professionals from nominated participating organisations who have administered the EPDS to an Aboriginal and/or Torres Strait Islander mother are considered eligible. Healthcare professionals 
include clinicians who have direct contact with clients or patients, such as (but not limited to) Aboriginal health workers, midwives, nurses, doctors, psychologists, psychiatrists. In total, there will be two participatory groups: Aboriginal mothers and healthcare professionals.

\section{Patient and public involvement}

This study will adopt a PPI approach for its design development and methodologies, but this is a research protocol paper, and thus patients were not involved at this stage.

\section{Sample size}

Sample size was determined on the basis of previous literature utilising thematic analyses. For small projects, Clarke and Braun ${ }^{63}$ suggested that six to 10 participants was the recommended number of participants if the project involved interviews. Furthermore, studies reported that after six interviews, thematic saturation was reached meaning that no further themes were found. ${ }^{6465}$ Another study sought 60 interviews, but discovered that thematic saturation was reached within 12 interviews, with broader themes apparent after six. ${ }^{66}$ Various sample sizes were suggested, but the general consensus from previous studies show that themes derived from six interviews will reach thematic saturation. This proposed project aims to gather a minimum of six Aboriginal mothers and a minimum of six healthcare professionals. In total, this will yield at least 12 participants, but researchers intend to continue inviting new participants until no additional themes surface. This number was justified by the amount of data available to demonstrate patterns while ensuring there is not too much data to manage.

There are complex reasons behind why a client may not be able to attend a scheduled perinatal appointment. And because this longitudinal research project is dependent on the client's follow-up attendance at different time points, it is a possibility that researchers may not be able to obtain the target sample size (six Aboriginal mothers). In this event, researchers are prepared to gather information by proxy. Assessing likely emotional responses by asking a proxy can substitute for a client's self-assessment. This proxy-patient assessment can be obtained by asking a proxy (from either Aboriginal mothers or fathers who were able to attend) to provide an assessment based on what they think their counterparts would respond. ${ }^{67}$

\section{Recruitment}

As part of routine practice, pregnant Aboriginal mothers will seek antenatal care from healthcare providers of their choice. Flyers (advertising the project) and a Participant Information Sheet will be first given directly to potential participants in the waiting room as an invitation to join the study. If mothers are interested in participating in this research project, they will be given an opportunity to clarify any concerns and enquiries on the next scheduled appointment with a healthcare professional. Mothers will be given the opportunity to provide a signed informed consent to participate in the research project during their next scheduled appointment, when the EPDS is routinely administered.

\section{Procedure}

Once consent has been obtained by participants, EPDS administration will be conducted when mothers attend their scheduled appointment to access routine antenatal care. The EPDS will be administrated again 6-8 weeks after childbirth when mothers are again scheduled for routine postnatal care. ${ }^{2568}$ Subsequent to each administration and completion of EPDS, Aboriginal cultural consultants will conduct one-on-one semistructured interviews with Aboriginal mothers. Interviews will involve participants reflecting on their understanding of perinatal mental health and their experience of the EPDS process.

At the final stage, the first author will conduct a further one-on-one semistructured interview with: (i) the Aboriginal cultural consultant to gain an additional perspective on participant experiences and (ii) healthcare providers to explore their experiences of administering the EPDS. As screening women for perinatal depression is part of routine care, referrals and arrangements are already established. However, should mothers participating in this study report that completing the EPDS questions and/or the interview thereafter had elicited emotional responses, the research team will arrange for referral to a service of their choice (ie, social workers, psychologists, Aboriginal heath workers) to ensure that they have access to safe support and follow-up care. All interviewers, both Aboriginal cultural consultants and the first author, will undergo training to standardise the interviewers as well as analysis. This, in addition to one-on-one interviews, will help strengthen the inter-reliability of the interviews.

\section{Outcome measures}

\section{Semistructured interviews}

Interview questions following completion of the EPDS will focus on exploring experiences of using and interacting with the EPDS (eg, 'What do you think the questions on the EPDS are asking?'; 'What do you think the rating scales mean? How do you decide what rating to give?'; 'Who do you think gets to see your responses to this survey?'; 'Did you know why you were being asked these questions? Are there other questions that might have given a better sense of how you are feeling?'). Opinions, experiences and preferences of healthcare professionals when administering the EPDS will be sought during interviews (eg, "Are there any questions that your clients seem particularly uncomfortable about or seem hesitant to answer?'; 'What about your approach in asking the questions did you think made your client feel comfortable/uncomfortable?'; 'Did you provide feedback to your client? If so, how was this done?'). Capturing healthcare professionals' views and experiences of using the EPDS can provide insight into the opinions and preferences of clinical staff, and how this could influence the application of EPDS. 


\section{Edinburgh Postnatal Depression Scale}

This 10-item self-report scale was designed to screen for postnatal depression in population community samples; with each item scored on a four-point scale, with a total score ranging from 0 to $30 .^{22}$ The items are primarily concerned with one's feelings referring to depressed mood, anhedonia, guilt, anxiety and suicidal ideation in the past week of their perinatal period.

The EPDS has extended to screening and detecting women who may be at risk for depression during both the antenatal and postnatal period (that together is known as the perinatal period). As previously indicated, internationally and nationally, the EPDS is considered gold standard for perinatal mental health screening and is a routine recommendation for screening mental health among mothers during pregnancy and 12 months following birth in many countries. Although research has demonstrated sound psychometric properties of the EPDS during the perinatal period, ${ }^{69}$ there are very few studies that include Aboriginal people in their samples.

\section{Data analyses}

Mixed-methods model

Mixed methods will be used. While the qualitative data (interview narratives) will undergo thematic content analyses, researchers will also look at individual EPDS scores in a qualitative way to see whether narratives reflect and/ or match with responses to the EPDS items. If there are discrepancies between or within individual case studies, qualitative narratives can lead to thematic conclusions and underlying process of test scores (ie, stress and fear associated with a new baby). So rather than quantifying the relationship between test score and criterion (traditional psychometric approach to validation), this adopted process-focused approach to validity highlights the nature of the tool and the context in which testing takes place.

Analysis of the two time periods (antenatally and postnatally) will also help us to better understand the process of changing mental health in the perinatal period and the potential for changing perceptions about screening and/ or about mental health. This will help researchers further understand and iteratively investigate any developmental aspects of perinatal mental health and also of the differential capacity of the EPDS versus interview self-report to identify these changes. ${ }^{70}$

\section{Thematic analyses}

Thematic content analysis of the interview transcripts will be conducted by the first author to identify thematic meanings underpinning of how mothers respond to the EPDS items and process. This analysis will review, examine and identify core common themes and patterns until no new themes surface or emerge; until thematic saturation is reached. ${ }^{59}$ Subsequently, to examine interrater reliability, an independent researcher will evaluate the codes and themes in a meaningful way to ensure there are no disagreements. This will reduce researcher bias by resolving any discrepancies and disagreements of themes in the process.

The qualitative analyses for this research project will draw on Charmaz's grounded theory that reconstructs rich experiences from interviews into emergent data categories as data is being collected, starting the chain of thematic development. ${ }^{71}$ The present study will also draw on most significant change (MSC) technique. The MSC technique is a participatory monitoring and evaluation technique that includes both research members and participants to be involved in deciding the sorts of changes to be recorded and analysed. ${ }^{72}$ Researchers and participants will look at significant changes, or the absence of them, in stories when comparing the antenatal and postnatal period. Lastly, thematic analysis will also draw on Maxwell's realist approach to qualitative research, which follows a model of ongoing research design as process rather than a linear relation. ${ }^{61}$ One of Maxwell's case studies of Inuit culture recognised the importance of integrating 'meaning' with cultural diversity, social contexts and behaviours as a valid way of generating explanations for a phenomena. ${ }^{61}{ }^{73}$ Parallels between Canadian Inuit and Aboriginal Australian culture are shown, so the role of qualitative research as process analysis stressed in Maxwell's case example can be applied to this protocol as it is compatible with the process-oriented validation framework.

Participant checking will be used to maintain a robust, high-quality dataset. Specifically, a summary of key themes from thematic and MSC analysis will be fed back to participants and nominated organisations for their preliminary input and feedback that could help future analyses in the subsequent EPDS administration. All participants will have access to current project outcomes and opportunities will be provided to communicate periodically with the research team.

\section{Cultural considerations}

This participatory mixed-method research project draws on elements from 'yarning' techniques, ${ }^{56-58}$ Green's thematic analyses, ${ }^{59}$ Maxwell's realist approach to qualitative research, ${ }^{61}{ }^{73}$ Charmaz's grounded theory ${ }^{71}$ and Dart and Davies' MSC $^{72}$ to support the development of a strong decolonising research methodology. ${ }^{74}$ These different forms of Aboriginal research methodologies also satisfy the fundamental principles of Rigney's Indigenist research. ${ }^{48}$ Adopting and implementing Indigenist research methodologies encompass collaborative partnership with Aboriginal communities as research partners, as it aims to share information in a reciprocal way; and most critical of all, showing sincere and genuine integrity towards the stories that are shared by Aboriginal women.

It is clear that the design and purpose of this study privileges cultural consideration by taking a less conventional approach of validation to progress the evidence base on perinatal mental health and well-being. Researchers will ensure cultural safety by continuing to prioritise consultation with Aboriginal community members and 
advisory groups, who will support cultural security and cultural guidance in this process. In addition, prior to commencing interviews with Aboriginal participants, interviewers will be trained in responding to participants' experiences in a culturally sensitive way.

\section{Data collection and monitoring}

Collecting data from Aboriginal Australian women during pregnancy is key in this research project. Data will be collected and managed in line with the National Statement on Ethical Conduct in Human Research of Australia to ensure the well-being and care of the woman and her unborn baby. ${ }^{75}$ As this is sensitive research involving Aboriginal and/Torres Strait Islander people, the research will be conducted in adherence to the core principles outlined in National Health and Medical Research Council (NHMRC) guidelines Values and Ethics: Guidelines on the Ethical Conduct of Research in Aboriginal and Torres Strait Islander Health Research. This is intended to ensure that the research team will build a trusting and supportive relationship with all participants while maintaining cultural responsiveness and cultural safety. ${ }^{76}$

All demographics, information derived from interviews and outcome data from questionnaires will be automatically de-identified using secure technology in accordance with the Murdoch IT research policy. The de-identified audio recordings will be made available for individual participants to verify and validate recordings, and then be transcribed by a researcher into key themes and subthemes. Participants will be advised that verbatim quotations from their interviews may be used in publications, but participants will not be identifiable. The research team will take full responsibility of all research data, which will be securely locked at the university facilities and on password-protected computers. Only relevant researchers can access this; research data will be retained and stored safely for a minimum of 5 years from date of publication.

\section{Ethics and dissemination}

The study foresees little to no risk to participants and their families. Written and signed informed consent to participate and be audio-recorded will be obtained from all participants. The Murdoch University's responsible conduct of research policy ensures that all research undertaken at Murdoch University complies with the Australian Code for the Responsible Conduct of Research. ${ }^{75}$

Findings of this project reflecting the input and feedback of EPDS users (Aboriginal mothers and healthcare professionals) will become recommendations for practice and policy change. We aim to disseminate the findings through regional and international conferences and in peer-reviewed journals.

\section{SIGNIFICANCE AND CONCLUSION}

Poor perinatal mental health has significant impacts for baby and mother and is more prevalent for Aboriginal than non-Aboriginal Australians. ${ }^{19}$ As the gold standard tool for routine use in Australia, it is crucial to evaluate the administration of the EPDS in the context of screening the perinatal mental health needs of Aboriginal mothers. While it has been acknowledged and noted that the EPDS should be used with caution when being administered with culturally and linguistically diverse populations, concerns have been raised that the EPDS may not be culturally compatible. ${ }^{36}$ There is currently no published data that adequately addresses this issue.

Although this study will pose no direct beneficial significance to participating Aboriginal mothers and their families, better understanding and gained insight into the experiences and perceptions of the EPDS screening process from the perspective of key stakeholders (Aboriginal mothers and healthcare professionals) can inform and guide optimal care and follow-up. This partnership with key stakeholders will provide a stronger foundation for future studies into the design of suitable perinatal mental health screening tools and processes. Most importantly, this research will be consistent with traditional philosophies of Aboriginal individuals and communities by recognising the complex methodological and ethical considerations in Aboriginal research and by maintaining a heavy emphasis on respecting and honouring Aboriginal voices through research. ${ }^{77}$

\section{Author affiliations}

${ }^{1}$ School of Psychology and Exercise Science, Murdoch University, Perth, Western Australia, Australia

${ }^{2}$ School of Health Professions, Murdoch University, Perth, Western Australia, Australia

${ }^{3}$ Ngangk Yira, Research Centre for Aboriginal Health \& Social Equity, Murdoch University, Perth, Western Australia, Australia

${ }^{4}$ School of Health in Social Science, University of Edinburgh, Scotland, UK

Acknowledgements The authors acknowledge that the Aboriginal and Torres Strait Islander peoples are the traditional owners of Australia. Although this paper is an independent project, it will assist in a larger study - the development of a new screening rubric for Aboriginal families. Authors would like to thank Aboriginal Advisory Group members of the larger study, Jayne Kotz, and all participating healthcare services who have given their support and approval to conduct this research protocol. The authors acknowledge the Western Australia Department of Health, Australian Institute of Health and Welfare and Australian Bureau of Statistics for their population statistics that have contributed to this paper.

Contributors AWC contributed to the conception and design of the work, and drafted the paper. CR contributed to the early development of the research project during the initial stages, and critically revised the paper. RM and PS supervised and managed its scientific direction, and critically revised the paper. RM is principal supervisor of the project, managing relationships. All authors have contributed to writing and reviewing, and agreed to this manuscript.

Funding The authors have not declared a specific grant for this research from any funding agency in the public, commercial or not-for-profit sectors.

Competing interests The reviewer has declared competing interests related to the submitted work article. There are no other relationships or conflicts to be declared.

Patient consent Not required.

Ethics approval Murdoch University (\#2017/125), Western Australian Aboriginal Health Ethics Committee (\#553), Women and Newborn Health Service (2014062EW) and St. John of God Health Care (\#1162).

Provenance and peer review Not commissioned; externally peer reviewed.

Open access This is an open access article distributed in accordance with the Creative Commons Attribution Non Commercial (CC BY-NC 4.0) license, which 
permits others to distribute, remix, adapt, build upon this work non-commercially, and license their derivative works on different terms, provided the original work is properly cited, appropriate credit is given, any changes made indicated, and the use is non-commercial. See: http://creativecommons.org/licenses/by-nc/4.0/.

\section{REFERENCES}

1. Lawrence E, Nylen K, Cobb RJ. Prenatal expectations and marital satisfaction over the transition to parenthood. J Fam Psychol 2007;21:155-64.

2. Australian Institute of Health and Welfare [AlHW]. Information Paper. Perinatal depression: data from the 2010 Australian National Infant Feeding Survey. Canberra, Australia: Australian Government, 2012. AlHW Cat. No. PHE 161. https://www.aihw.gov.au/getmedia/ 80df038a-4a03-4214-beca-cfd4b0ac6a43/14496.pdf.aspx?inline= true. (accessed 28 Aug 2017).

3. Halbreich U, Karkun S. Cross-cultural and social diversity of prevalence of postpartum depression and depressive symptoms. $J$ Affect Disord 2006;91:97-111.

4. Markwick A, Ansari Z, Sullivan M, et al. Inequalities in the social determinants of health of Aboriginal and Torres Strait Islander People: a cross-sectional population-based study in the Australian state of Victoria. Int J Equity Health 2014;13:91.

5. Jorm AF, Bourchier SJ, Cvetkovski S, et al. Mental health of Indigenous Australians: a review of findings from community surveys. Med J Aust 2012;196:118-21.

6. Australian Institute of Health and Welfare [AIHW]. The health and welfare of Australia's Aboriginal and Torres Strait Islander peoples 2015. Canberra, Australia: Australian Government. AlHW Cat. No. IHW 147. https://www.aihw.gov.au/getmedia/584073f7-041e-4818 9419-39f5a060b1aa/18175.pdf.aspx?inline=true. (accessed 28 Aug 2017)

7. Barbour B. Addressing Aboriginal disadvantage: The need to do things differently. Special Report to Parliament under s 31 of the Ombudsman Act 1974. Sydney, Australia: New South Wales Ombudsman, 2011. https://www.ombo.nsw.gov.au/_data/assets/ pdf_file/0012/3342/SR_Aboriginal-disadvantage-report.pdf. (accessed 28 Aug 2017).

8. Eley D, Hunter K, Young L, et al. Tools and methodologies for investigating the mental health needs of Indigenous patients: it's about communication. Australas Psychiatry 2006;14:33-7.

9. Eberhard-Gran M, Eskild A, Tambs K, et al. Depression in postpartum and non-postpartum women: prevalence and risk factors. Acta Psychiatr Scand 2002;106:426-33.

10. World Health Organisation [WHO]. Maternal mental health: WHO, 2018. http://www.who.int/mental_health/maternal-child/maternal mental_health/en/. (accessed 3 Jan 2018).

11. Fisher J, Cabral de Mello M, Patel V, et al. Prevalence and determinants of common perinatal mental disorders in women in low- and lower-middle-income countries: a systematic review. Bull World Health Organ 2012;90:139-49.

12. Kildea S, Kruske S, Barclay L, et al. 'Closing the Gap': how maternity services can contribute to reducing poor maternal infant health outcomes for Aboriginal and Torres Strait Islander women. Rural Remote Health 2010;10:1383

13. Australian Institute of Health and Welfare [AIHW]. Australia's mother and babies 2004. Sydney, Australia: AlHW National Perinatal Statistics Unit, 2006. AlHW cat. no. PER 3. http://www.aihw.gov.au/ WorkArea/DownloadAsset.aspx?id=6442458950. (accessed 28 Aug 2017).

14. Australian Health Ministers' Advisory Council [AHMAC]. Aboriginal and Torres Strait Islander Health Performance Framework 2017 Report. Canberra, Australia: AHMAC Australian Government, 2017. https://www.pmc.gov.au/sites/default/files/publications/2017-healthperformance-framework-report.pdf. (accessed 28 Aug 2017).

15. Department of Health [DoH]. Perinatal, infant and maternal mortality in Western Australia, 2006-2010 Maternal mental health. Perth, Australia: DoH State of Western Australia. Statistical Series/95 ISSN 0816-88. http://www.healthywa.wa.gov.au/ /media/Files/Corporate/ Reports\%20and\%20publications/Perinatal\%20infant\%20and\% 20maternal/Perinatal_Infant_Maternal_Mortality_WA_2006-2010. ashx. (accessed 28 Aug 2017).

16. Sayers S, Boyle J. Indigenous perinatal and neonatal outcomes: a time for preventive strategies. J Paediatr Child Health 2010;46:475-8.

17. Australian Institute of Health and Welfare [AIHW]. Indigenous mothers and their babies, Australia 2001-2004. AlHW Perinatal Statistics Series Number 19. Canberra, Australia: Australian Government, 2017. AIHW cat. no. PER 38. http://www.aihw.gov.au/WorkArea/ DownloadAsset.aspx?id=6442458951. (accessed 28 Aug 2017).
18. Ferguson-Hill S. Promoting perinatal mental health wellness in Aboriginal and Torres Strait Islander communities. In: Dudgeon P, Milroy $\mathrm{H}$, Walker $\mathrm{R}$ second, eds. Working Together: Aboriginal and Torres Strait Islander Mental Health and Wellbeing Principles and Practice. Canberra, Australia, 2014:223-42.

19. beyondblue.org.au. Clinical practice guidelines for depression and related disorders - anxiety, bipolar disorder and puerperal psychosis - in the perinatal period A Comprehensive Systematic Literature Review. Australia: beyondblueAustralia's National Depression Initiative, 2009. https://www.beyondblue.org.au/docs/default-source/ 8.-perinatal-documents/bw0104-perinatal-slr-tools-q1-14.pdf? sfvrsn=2. (accessed 28 Aug 2017).

20. Marriott R, Ferguson-Hill S. Perinatal and infant mental health and wellbeing. In: Dudgeon P, Milroy $\mathrm{H}$, Walker R second, eds. Working Together: Aboriginal and Torres Strait Islander Mental Health and Wellbeing Principles and Practice. Canberra, Australia, 2014:337-53.

21. Patel V, Rahman A, Jacob KS, et al. Effect of maternal mental health on infant growth in low income countries: new evidence from South Asia. BMJ 2004;328:820-3.

22. Fox S, Smith C, Emerson L. Australian Research Alliance for Children and Youth, Lowitja Institute. Good beginnings: getting it right in the early years: review of the evidence on the importance of a healthy start to life and on interventions to promote good beginnings. Melbourne (Carlton), Australia: The Lowitja Institute, 2015.

23. Sivak L, Arney F, Lewig K. Department of Innovation Industry, Science and Research; University of AdelaideAustralian Centre for Child Protection (ACCP). A pilot exploration of a family home visiting program for families of Aboriginal and Torres Strait Islander children: perspectives of parents of Aboriginal children and organisational considerations. Adelaide, Australia, 2008. http://search.ror.unisa.edu. au/record/UNISA_ALMA11143309610001831/media/digital/open/ 9915911179001831/12143309600001831/13143306010001831/pdf. (accessed 28 Aug 2017).

24. Cox JL, Holden JM, Sagovsky R. Detection of postnatal depression. development of the 10-item edinburgh postnatal depression scale. Br J Psychiatry 1987;150:782-6.

25. Department of Health [DoH] Community Health. Birth to School Entry, Child and Adolescent Health Service [CAHS] State of Western Australia. Edinburgh Postnatal Depression Scale (EPDS) - guidelines for use community health manual. Perth, Australia, 2007. http://www. pmh.health.wa.gov.au/general/CACH/docs/manual/3\%20Birth\% 20to\%20School\%20Entry/3.8/3.8.4\%20Birth\%20to\%20School\% 20Entry\%20Resources\%20-\%20Edinburgh\%20Postnatal.pdf. (accessed 28 Aug 2017).

26. Austin MP, Middleton P, Reilly NM, et al. Detection and management of mood disorders in the maternity setting: the Australian Clinical Practice Guidelines. Women Birth 2013;26:2-9.

27. Austin MP, Lumley J. Antenatal screening for postnatal depression: a systematic review. Acta Psychiatr Scand 2003;107:10-17.

28. Cox J, Holden J. Perinatal mental health: a guide to the Edinburgh Postnatal Depression Scale (EPDS. London, UK: RCPsych Publications, 2003.

29. Austin M-P, Highet N. Expert Working GroupCentre of Perinatal Excellence. Effective Mental Health Care in the Perinatal Period: Australian Clinical Practice Guideline. Melbourne, Australia: Australian Clinical Practice Guideline, 2017. http://cope.org.au/ about/review-of-new-perinatal-mental-health-guidelines/. (accessed 27 Aug 2017).

30. Hayes BA, Campbell A, Buckby B, et al. The interface of mental and emotional health and pregnancy in urban indigenous women: Research in progress. Infant Ment Health J 2010;31:277-90.

31. Brown R. Australian Indigenous mental health. Aust N Z J Ment Health Nurs 2001;10:33-41.

32. McDonald H. East Kimberley concepts of health and illness: a contribution to intercultural health programs in Northern Australia. : Australian Aboriginal Studies, 2006:2: 86-97.

33. Vicary DA, Bishop BJ. Western psychotherapeutic practice: Engaging Aboriginal people in culturally appropriate and respectful ways. Aust Psychol 2005;40:8-19.

34. Vicary D, Westerman T. That's just the way he is': Some implications of Aboriginal mental health beliefs. Australian e-Journal for the Advancement of Mental Health 2004;3:103-12.

35. WA Country Health Service [WACHS] and WA Perinatal Mental Health Unit [WAPMHU]. WACHS Staffing Needs Analysis. Perinatal_ Depression_Scoping_report. Perth, Australia: Department of Health [DoH], 2017. https://www.kemh.health.wa.gov.au/ /media/Files/ Hospitals/WNHS/Our\%20Services/State-wide\%20Services/WHSP/ Perinatal Depression Scoping report.pdf.

36. Kotz J, Munns A, Marriott R, et al. Perinatal depression and screening among Aboriginal Australians in the Kimberley. Contemp Nurse 2016;52:42-58. 
37. WA Perinatal Mental Health Unit [WAPMHU]Women and Newborn Health Service. Aboriginal Perinatal Service Expansion: Baseline Evaluation Report. Perth, Australia: Department of Health [DoH] State of Western Australia, 2008. (accessed 28 Aug 2017).

38. Atkinson J. Trauma trails, recreating song lines: the transgenerational effects of trauma in Indigenous Australia. North Melbourne, Australia: Spinifex Press, 2002.

39. Garvey D. Australian Indigenous Healthbulletin. A review of the social and emotional wellbeing of Indigenous Australian peoples - considerations, challenges and opportunities (peer reviewed). 2008;8:288KB.

40. Koolmatrie J, Williams R. Unresolved grief and the removal of indigenous Australian Children. Aust Psychol 2000;35:158-66.

41. Gaynes BN, Gavin N, Meltzer-Brody S, et al. Perinatal Depression: Prevalence, Screening Accuracy, and Screening Outcomes. Rockville, USA: Agency for Healthcare Research and Quality, 2005:30. Summary, Evidence Report/Technology Assessment: Number 119. http://www.ahrq.gov/clinic/epcsums/ peridepsum.htm. (accessed 28 Aug 2017).

42. Campbell A, Hayes B, Buckby B. Aboriginal and Torres Strait Islander women's experience when interacting with the Edinburgh Postnatal Depression Scale: a brief note. Aust J Rural Health 2008;16:124-31.

43. Parliament of Australia. Chapter 2: The role of Indigenous languages. House of Representatives Standing Committee on Aboriginal and Torres Strait Islander Affairs. Canberra, Australia, 2012. https:// www.aph.gov.au/Parliamentary_Business/Committees/House_of_ representatives_Committees?url=/atsia/languages2/report.htm. (accessed 21 Nov 2017).

44. Marley JV, Kotz J, Engelke C, et al. Validity and acceptability of kimberley mum's mood scale to screen for perinatal anxiety and depression in remote aboriginal health care settings. PLoS One 2017;12:e0168969.

45. Australia CDepartment of the Prime Minister and Cabinet. Closing the Gap Prime Minister's Report 2018: Australian Government, 2018. https://closingthegap.pmc.gov.au/sites/default/files/ctg-report-2018. pdf.

46. Howe A, Mathie E, Munday D, et al. Learning to work together lessons from a reflective analysis of a research project on public involvement. Res Involv Engagem 2017;3.

47. Bornstein RF. Toward a process-focused model of test score validity: improving psychological assessment in science and practice. Psychol Assess 2011;23:532-44.

48. Rigney L-I. Internationalization of an indigenous anticolonial cultural critique of research methodologies: a guide to indigenist research methodology and its principles. Wicazo Sa Review 1999;14:109-13.

49. Australian Institute of Health and Welfare [AlHW]. Nursing and Midwifery Workforce 2015: Australian Government; 2016. Web Report., 2017. https://www.aihw.gov.au/reports/workforce/nursingand-midwifery-workforce-2015/contents/who-are-nurses-andmidwives

50. Barwick MA, Peters J, Boydell K. Getting to uptake: do communities of practice support the implementation of evidence-based practice? $J$ Can Acad Child Adolesc Psychiatry 2009;18:16-29.

51. National Health and Medical Research Council [NHMRC]. Cultural Competency in Health: A guide for policy, partnerships and participation. Canberra, Australia: Commonwealth of Australia, 2006. https://www.nhmrc.gov.au/ files nhmrc/publications/attachments/ hp19.pdf. (access 14 Dec 2017).

52. Hayward K, Winch J. 'Doing it our way': can cultural traditions survive in universities? New Doctor 1998;70:25-7.

53. Creswell JW, Plano Clark VL. Designing and conducting mixed methods research. Thousand Oaks, California, USA: SAGE Publications, 2006.

54. Denzin NK. The research act: a theoretical introduction to sociological methods. NY, USA: McGraw-Hill, 1978.

55. Denzin NK. The research act: a theoretical introduction to sociological methods. London, UK; Somerset, N.J: Aldine Transaction, 2009.

56. Lin I, Green C, Bessarab D. 'Yarn with me': applying clinical yarning to improve clinician-patient communication in Aboriginal health care. Aust J Prim Health 2016;22:377-82.
57. Geia LK, Hayes B, Usher K. Yarning/Aboriginal storytelling: Towards an understanding of an Indigenous perspective and its implications for research practice. Contemp Nurse 2013;46:13-17.

58. Pia E. Narrative Therapy and Peacebuilding. Journal of Intervention and Statebuilding 2013;7:476-91.

59. Green J, Willis K, Hughes E, et al. Generating best evidence from qualitative research: the role of data analysis. Aust $N Z J$ Public Health 2007;31:545-50.

60. DeSantis L, Ugarriza DN. The concept of theme as used in qualitative nursing research. West $J$ Nurs Res 2000;22:351-72.

61. Maxwell JA. A realist approach for qualitative research. Thousand Oaks, CA: SAGE Publications, 2012.

62. Cameron R. A sequential mixed model research design: Design, analytical and display issues. Int $J$ Mult Res Approaches 2009;3:140-52.

63. Clarke V, Braun V. Successful qualitative research: a practical guide for beginners. London, UK: SAGE Publications, 2013.

64. Isman E, Ekéus C, Berggren V. Perceptions and experiences of female genital mutilation after immigration to Sweden: an explorative study. Sex Reprod Healthc 2013;4:93-8.

65. Isman E, Mahmoud Warsame A, Johansson A, et al. Midwives' experiences in providing care and counselling to women with female genital mutilation (fgm) related problems. Obstet Gynecol Int 2013;2013:1-9.

66. Guest G, Bunce A, Johnson L. How many interviews are enough? an experiment with data saturation and variability. Field Methods 2006;18:59-82.

67. Pickard AS, Knight SJ. Proxy evaluation of health-related quality of life: a conceptual framework for understanding multiple proxy perspectives. Med Care 2005;43:493-9.

68. Department of Health [DoH] Child and Adolescent Health Service [CAHS]. Policy: Aboriginal Child Health. Perth, Australia: Country Health Service (WACHS) State of Western Australia; 2012. Child Health Manual, 2017. http://www.rph.wa.gov.au/ /media/Files/ Corporate/general\%20documents/CACH/CHM/CACH.CHSH.Abor iginalChildHealthPolicy.ashx.

69. Boyd RC, Le HN, Somberg R. Review of screening instruments for postpartum depression. Arch Womens Ment Health 2005;8:141-53.

70. Wisdom J, Creswell J. Mixed Methods: Integrating Quantitative and Qualitative Data Collection and Analysis While Studying PatientCentered Medical Home Models. Rockville, USA: Agency for Healthcare Research and Quality, US Department of Health and Human Services, 2017. AHRQ Publication No. 13-08-EF. https:// pcmh.ahrq.gov/page/mixed-methods-integrating-quantitative-andqualitative-data-collection-and-analysis-while.

71. Charmaz K. Grounded theory in the $21^{\text {st }}$ century: A qualitative method for advancing social justice Research. In: Denzin N, Lincoln Y third, eds. Handbook Of Qualitative Research, Thousand Oaks, CA SAGE Publications, 2005

72. Dart J, Davies R. A dialogical, story-based evaluation tool: the most significant change technique. Am J Eval 2003;24:137-55.

73. Kuusela P, Joseph A. Maxwell Book review: Realist Approach for Qualitative Research. . London: SAGE. Qualitative Sociology Review, 2011:8. 90-2. http://www.qualitativesociologyreview.org/ENG/ archive_eng.php.

74. Smith LT. Decolonizing methodologies: research and indigenous peoples. 2nd ed. Dunedin, N.Z; London; UK; New York, USA: Zed Books Ltd, 2012.

75. National Health and Medical Research Council [NHMRC]. National statement on ethical conduct in human research. Canberra, Australia: NHMRC Australian Government. https://www.nhmrc.gov. au/_files_nhmrc/publications/attachments/e72_national_statement_ may_2015_150514_a.pdf. (accessed 28 Aug 2017).

76. National Health and Medical Research Council [NHMRC]. Values and ethics: Guidelines on ethical conduct in Aboriginal and Torres Strait Islander health research. Canberra, Australia: NHMRC Australian Government. https://www.nhmrc.gov.au/ files nhmrc/publications/ attachments/e52.pdf. (access 28 Aug 2017)

77. Smith LT. On tricky ground: Researching the native in the age of uncertainty. In: Denzin NK, Lincoln YS third, eds. The Sage Handbook of Qualitative ResearchThousand Oaks, CA: SAGE Publications, 2005:85-108. 